\title{
Wigner's phase-space function and atomic structure: II. Ground states for closed-shell atoms
}

Springborg, Michael; Dahl, Jens Peder

Published in:

Physical Review A

Link to article, DOI:

10.1103/PhysRevA.36.1050

Publication date:

1987

Document Version

Publisher's PDF, also known as Version of record

Link back to DTU Orbit

Citation (APA):

Springborg, M., \& Dahl, J. P. (1987). Wigner's phase-space function and atomic structure: II. Ground states for closed-shell atoms. Physical Review A, 36(3), 1050-1062. https://doi.org/10.1103/PhysRevA.36.1050

\section{General rights}

Copyright and moral rights for the publications made accessible in the public portal are retained by the authors and/or other copyright owners and it is a condition of accessing publications that users recognise and abide by the legal requirements associated with these rights.

- Users may download and print one copy of any publication from the public portal for the purpose of private study or research.

- You may not further distribute the material or use it for any profit-making activity or commercial gain

- You may freely distribute the URL identifying the publication in the public portal 


\title{
Wigner's phase-space function and atomic structure: II. Ground states for closed-shell atoms
}

\author{
Michael Springborg \\ Chemistry Department B, Technical University of Denmark, Bld. 301, DK 2800 Lyngby, Denmark \\ and Nordisk Institut for Teoretisk Atomfysik (NORDITA), Blegdamsvej 17, DK 2100 København $\emptyset$, Denmark* \\ Jens Peder Dahl \\ Chemistry Department B, Technical University of Denmark, Bld. 301, DK 2800 Lyngby, Denmark
}

(Received 29 December 1986)

\begin{abstract}
We present formulas for reduced Wigner phase-space functions for atoms, with an emphasis on the first-order spinless Wigner function. This function can be written as the sum of separate contributions from single orbitals (the natural orbitals). This allows a detailed study of the function. Here we display and analyze the function for the closed-shell atoms helium, beryllium, neon, argon, and zinc in the Hartree-Fock approximation. The quantum-mechanical exact results are compared with those obtained with the approximate Thomas-Fermi description of electron densities in phase space.
\end{abstract}

\section{INTRODUCTION}

Already in the 1920s a statistical theory giving an approximate description of electron densities in phase space (the direct product of position and momentum space) was proposed by Thomas ${ }^{1}$ and Fermi. ${ }^{2}$ This theory, with later improvements (see, e.g., Ref. 3), is still very useful (for a recent review, see, e.g., Ref. 4).

A few years later an exact transformation from a wave function in either position or momentum space to a function in phase space, which in some sense can be interpreted as an electron density in phase space (see Sec. II), was introduced by Wigner. ${ }^{5}$ At about the same time, Weyl ${ }^{6}$ discussed the correspondence between quantummechanical operators and dynamical functions in phase space. Later developments ${ }^{7,8}$ led to a synthesis of Weyl's and Wigner's approaches and subsequently to a welldefined representation of quantum mechanics. A good introduction to this representation is due to de Groot and Suttorp. ${ }^{9}$

The phase-space representation has, for instance, found applications in quantum-mechanical studies of transport processes, ${ }^{10}$ radiation, ${ }^{11}$ and electronic devices, ${ }^{12}$ as well as in optics, ${ }^{13}$ and in molecular ${ }^{14}$ and nuclear dynamics. ${ }^{15,16}$ The dynamical applications are often combined with a classical description of the time evolution since it is too complicated to consider the full quantum-mechanical description of the time evolution (for a recent example, see the calculation of the photodissociation of $\mathrm{CH}_{3} \mathrm{I}$ by Henriksen ${ }^{17}$ ). Recent reviews on the phase-space representation of quantum mechanics discuss the general formalism, ${ }^{18,19}$ the semiclassical limit, ${ }^{20}$ and applications in quantum collision theory. ${ }^{21}$

Much insight may be gained by performing detailed studies of the Wigner functions for stationary states of quantum-mechanical systems. Considerable attention has been paid to one-dimensional systems like the harmonic oscillator, ${ }^{7,22-25}$ a particle in a linear potential, ${ }^{25,26}$ the free particle, ${ }^{25}$ and the Morse oscillator. ${ }^{27,28}$ However, there exist only few works on the phase-space function for atomic and molecular systems in the literature, namely two on the hydrogen atom, ${ }^{29,30}$ including the first paper in this series, ${ }^{29}$ and one by one of us on the LiH molecule. ${ }^{31}$ Therefore, it will be useful to extend the analysis of Wigner's phase-space function to atoms with more electrons. Within the Hartree-Fock approximation the formulas become particularly simple and the analysis more elucidating, so in the present paper we focus on closedshell atoms for which the Hartree-Fock approximation is reasonable. Related approaches have been presented by Thakkar $^{32}$ and Weyrich ${ }^{33}$ at two conferences.

The outline of the paper is as follows: In Sec. II we introduce the full Wigner function for an $N$-electron system and define reduced spinless phase-space functions. In Sec. III we describe how we have calculated the one-electron phase-space function for closed-shell atoms within the Hartree-Fock approximation. We shall compare our results with the approximations inherent in and the results predicted by the simplest Thomas-Fermi theory, so in Sec. IV we review this theory briefly. The succeeding sections are devoted to the results on atoms with only filled $s$ shells (helium and beryllium, Sec. V), atoms with filled $s$ and $p$ shells (neon and argon, Sec. VI), and an atom with filled $s, p$, and $d$ shells (zinc, Sec. VII). Finally, Sec. VIII is the conclusion. In an appendix we give some details of the derivation of the formulas used in calculating the Wigner function and present the final formulas.

\section{WIGNER PHASE-SPACE FUNCTIONS}

Let $|\Psi\rangle$ be the normalized state vector describing a stationary state of an $\mathrm{N}$-electron atom or molecule, and let $\Psi\left(\mathbf{r}_{1}, \ldots, \mathbf{r}_{N} ; \sigma_{1}, \ldots, \sigma_{N}\right)$ be the representative wave function in the combined position and spin space. The corresponding wave function in the combined momentum and spin space, $\Phi\left(\mathbf{p}_{1}, \ldots, \mathbf{p}_{N} ; \sigma_{1}, \ldots, \sigma_{N}\right)$, is the $3 N$ dimensional Fourier transform of $\Psi$, and vice versa. The full Wigner function, which combines the coordinate and momentum characteristics in a single picture, is ${ }^{5,9}$ 


$$
\begin{aligned}
& \Gamma\left(\mathbf{r}_{1}, \ldots, \mathbf{r}_{N} ; \mathbf{p}_{1}, \ldots, \mathbf{p}_{N} ; \sigma_{1}, \ldots, \sigma_{N} ; \sigma_{1}^{\prime}, \ldots, \sigma_{N}^{\prime}\right) \\
& =N !(2 / h)^{3 N} \int \cdots \int d \mathbf{q}_{1} \cdots d \mathbf{q}_{N} \Psi\left(\mathbf{r}_{1}+\mathbf{q}_{1}, \ldots, \mathbf{r}_{N}+\mathbf{q}_{N} ; \sigma_{1}, \ldots, \sigma_{N}\right) \Psi^{*}\left(\mathbf{r}_{1}-\mathbf{q}_{1}, \ldots, \mathbf{r}_{N}-\mathbf{q}_{N} ; \sigma_{1}^{\prime}, \ldots, \sigma_{N}^{\prime}\right) \\
& \times \exp \left[-\frac{2 i}{\hbar}\left(\mathbf{p}_{1} \cdot \mathbf{q}_{1}+\cdots+\mathbf{p}_{N} \cdot \mathbf{q}_{N}\right)\right] .
\end{aligned}
$$

It is seen to be a certain Fourier transform of the full density matrix

$$
\gamma_{N}\left(\mathbf{r}_{1}, \ldots, \mathbf{r}_{N} ; \mathbf{r}_{1}^{\prime}, \ldots, \mathbf{r}_{N}^{\prime} ; \sigma_{1}, \ldots, \sigma_{N} ; \sigma_{1}^{\prime}, \ldots, \sigma_{N}^{\prime}\right)=N ! \Psi\left(\mathbf{r}_{1}, \ldots, \mathbf{r}_{N} ; \sigma_{1}, \ldots, \sigma_{N}\right) \Psi^{*}\left(\mathbf{r}_{1}^{\prime}, \ldots, \mathbf{r}_{N}^{\prime} ; \sigma_{1}^{\prime}, \ldots, \sigma_{N}^{\prime}\right)
$$

The amount of information contained in the full density matrix or, equivalently, in the full Wigner function is very large, and for most purposes less is needed. This has led to the definition of reduced density matrices ${ }^{34-37}$ and, similarly, reduced Wigner functions. ${ }^{7}$ These quantities are in general spin dependent, but here we shall consider only the spinless quantities, ${ }^{37}$ defined by the following relations, with $n=1, \ldots, N$ :

$$
\begin{aligned}
\rho_{n}\left(\mathbf{r}_{1}, \ldots, \mathbf{r}_{n} ; \mathbf{r}_{1}^{\prime}, \ldots, \mathbf{r}_{n}^{\prime}\right) & \\
=N(N+1) \cdots(N-n+1) \int \cdots \int d \mathbf{r}_{n+1} \cdots d \mathbf{r}_{N} \int \cdots \int & \sigma_{1} \cdots d \sigma_{N} \\
& \times \Psi\left(\mathbf{r}_{1}, \ldots, \mathbf{r}_{n}, \mathbf{r}_{n+1}, \ldots, \mathbf{r}_{N} ; \sigma_{1}, \ldots, \sigma_{N}\right) \\
& \times \Psi^{*}\left(\mathbf{r}_{1}^{\prime}, \ldots, \mathbf{r}_{n}^{\prime}, \mathbf{r}_{n+1}, \ldots, \mathbf{r}_{N} ; \sigma_{1}, \ldots, \sigma_{N}\right)
\end{aligned}
$$

and

$$
\begin{aligned}
& f_{n}\left(\mathbf{r}_{1}, \ldots, \mathbf{r}_{n} ; \mathbf{p}_{1}, \ldots, \mathbf{p}_{n}\right) \\
& \quad=(2 / h)^{3 n} \int \cdots \int d \mathbf{q}_{1} \cdots d \mathbf{q}_{n} \rho_{n}\left(\mathbf{r}_{1}+\mathbf{q}_{1}, \ldots, \mathbf{r}_{n}+\mathbf{q}_{n} ; \mathbf{r}_{1}-\mathbf{q}_{1}, \ldots, \mathbf{r}_{n}-\mathbf{q}_{n}\right) \exp \left(-\frac{2 i}{\hbar}\left(\mathbf{p}_{1} \cdot \mathbf{q}_{1}+\cdots+\mathbf{p}_{n} \cdot q_{n}\right)\right) .
\end{aligned}
$$

The quantities for successive $n$ values are simply related; thus

$$
\int d \mathbf{r}_{n+1} \rho_{n+1}\left(\mathbf{r}_{1}, \ldots, \mathbf{r}_{n}, \mathbf{r}_{n+1} ; \mathbf{r}_{1}^{\prime}, \ldots, \mathbf{r}_{n}^{\prime}, \mathbf{r}_{n+1}\right)=(N-n) \rho_{n}\left(\mathbf{r}_{1}, \ldots, \mathbf{r}_{n} ; \mathbf{r}_{1}^{\prime}, \ldots, \mathbf{r}_{n}^{\prime}\right)
$$

and

$$
\int d \mathbf{r}_{n+1} d \mathbf{p}_{n+1} f_{n+1}\left(\mathbf{r}_{1}, \ldots, \mathbf{r}_{n}, \mathbf{r}_{n+1} ; \mathbf{p}_{1}, \ldots, \mathbf{p}_{n}, \mathbf{p}_{n+1}\right)=(N-n) f_{n}\left(\mathbf{r}_{1}, \ldots, \mathbf{r}_{n} ; \mathbf{p}_{1}, \ldots, \mathbf{p}_{n}\right) .
$$

Let us now consider a symmetric $N$-electron operator, built from the quantum-mechanical position and momentum operators $\mathbf{R}_{1}, \ldots, \mathbf{R}_{N}$ and $\mathbf{P}_{1}, \ldots, \mathbf{P}_{N}$, i.e.,

$$
A_{n}^{N}=\frac{1}{n !} \sum_{i_{1}}^{\prime} \cdots \sum_{i_{n}}^{\prime} A_{n}\left(\mathbf{R}_{i_{1}}, \ldots, \mathbf{R}_{i_{n}} ; \mathbf{P}_{i_{1}}, \ldots, \mathbf{P}_{i_{n}}\right)
$$

where each of the indices $i_{1}, \ldots, i_{n}$ scan the numbers $1, \ldots, N$, but with terms for which two or more indices are equal omitted (as indicated by the primes). Using the condition that $\Psi$ be an antisymmetric wave function, it is then easy to derive the standard expression

$$
\left\langle\Psi\left|A_{n}^{N}\right| \Psi\right\rangle=\frac{1}{n !} \int \cdots \int d \mathbf{r}_{1} \cdots d \mathbf{r}_{n}\left[A_{n}\left(\mathbf{R}_{1}, \ldots, \mathbf{R}_{n} ; \mathbf{P}_{1}, \ldots, \mathbf{P}_{n}\right) \rho_{n}\left(\mathbf{r}_{1}, \ldots, \mathbf{r}_{n} ; \mathbf{r}_{1}^{\prime}, \ldots, \mathbf{r}_{n}^{\prime}\right)\right]_{\mathbf{r}^{\prime}=\mathbf{r}},
$$

where $\mathbf{r}^{\prime}=\mathbf{r}$ indicates that the primes should be removed from $\mathbf{r}_{1}^{\prime}, \ldots, \mathbf{r}_{n}^{\prime}$ before the integrations are performed.

Similarly, we get

$$
\left\langle\Psi\left|A_{n}^{N}\right| \Psi\right\rangle=\frac{1}{n !} \int \cdots \int d \mathbf{r}_{1} \cdots d \mathbf{r}_{n} d \mathbf{p}_{1} \cdots d \mathbf{p}_{n} a_{n}\left(\mathbf{r}_{1}, \ldots, \mathbf{r}_{n} ; \mathbf{p}_{1}, \ldots, \mathbf{p}_{n}\right) f_{n}\left(\mathbf{r}_{1}, \ldots, \mathbf{r}_{n} ; \mathbf{p}_{1}, \ldots, \mathbf{p}_{n}\right)
$$

where the dynamical phase-space function $a_{n}$ is the Weyl transform ${ }^{6,24,38}$ of the operator $A_{n} . a_{n}$ and $A_{n}$ have the same functional form whenever $a_{n}$ only depends on the coordinates or the momenta, but may otherwise differ by terms in $\hbar$. A simple scheme for determining $A_{n}$ when $a_{n}$ is a polynomial in the r's and the p's is due to McCoy. ${ }^{9,29,39}$ However, we may always write

$$
A_{n}(1, \ldots, n)=(2 / h)^{3 n} \int \cdots \int d \mathbf{r}_{1} \cdots d \mathbf{r}_{n} d \mathbf{p}_{1} \cdots d \mathbf{p}_{n} a_{n}\left(\mathbf{r}_{1}, \ldots, \mathbf{r}_{n} ; \mathbf{p}_{1}, \ldots, \mathbf{p}_{n}\right) \Pi_{n}\left(\mathbf{r}_{1}, \ldots, \mathbf{r}_{n} ; \mathbf{p}_{1}, \ldots, \mathbf{p}_{n}\right)
$$


where

$\Pi_{n}\left(\mathbf{r}_{1}, \ldots, \mathbf{r}_{n} ; \mathbf{p}_{1}, \ldots, \mathbf{p}_{n}\right)=1 /(2 h)^{3 n} \int \cdots \int d \mathbf{u}_{1} \cdots d \mathbf{u}_{n} d \mathbf{v}_{1} \cdots d \mathbf{v}_{n} \exp \left[\frac{i}{\hbar} \sum_{k}\left[\left(\mathbf{r}_{k}-\mathbf{R}_{k}\right) \cdot \mathbf{u}_{k}+\left(\mathbf{p}_{k}-\mathbf{P}_{k}\right) \cdot \mathbf{v}_{k}\right]\right)$

is the inversion operator with respect to the phase-space point $\left(\mathbf{r}_{1}, \ldots, \mathbf{r}_{n} ; \mathbf{p}_{1}, \ldots, \mathbf{p}_{n}\right) .{ }^{24,38,40-42}$ Using this operator, but with running indices on $\mathbf{R}$ and $\mathbf{P}$, we may also show that

$$
\begin{aligned}
f_{n}\left(\mathbf{r}_{1}, \ldots,\right. & \left.\mathbf{r}_{n} ; \mathbf{p}_{1}, \ldots, \mathbf{p}_{n}\right) \\
& =(2 / h)^{3 n}\left\langle\Psi\left|\Pi_{n}^{N}\left(\mathbf{r}_{1}, \ldots, \mathbf{r}_{n} ; \mathbf{p}_{1}, \ldots, \mathbf{p}_{n}\right)\right| \Psi\right\rangle,
\end{aligned}
$$

in the notation of Eq. (7).

Equation (9) shows that

$$
(1 / n !) f_{n}\left(\mathbf{r}_{1}, \ldots, \mathbf{r}_{n} ; \mathbf{p}_{1}, \ldots, \mathbf{p}_{n}\right)
$$

plays the role of a phase-space distribution function in an operational sense, but although it is always real, it may be locally negative.

In the above discussion we have derived the Wigner function from the position space density matrices. We might, however, equally well have used the momentumspace density matrices. The only difference is that if the $\Psi$ 's on the right-hand side of Eq. (1) are replaced by $\Phi$ 's, then the sign must be changed in the argument of the exponential function. Similar remarks apply to the other equations above.

We also want to point out that it is possible to construct a set of coupled phase-space differential equations which the full Wigner function (1) must satisfy. ${ }^{25}$ These differential equations are in general of infinitely high order and therefore difficult to solve. Accordingly, they have so far only been solved directly for some one-dimensional systems, ${ }^{25,43}$ including the free particle, a particle in a linear potential, and the harmonic oscillator. In addition they have been used in a discussion of the BohrHeisenberg correspondence principle. ${ }^{44}$ In all these cases the equations become of finite order.

The easiest way of calculating the phase-space function for atomic and molecular systems is to apply (4) with the density matrix obtained using some of the standard methods of quantum chemistry. This is the way we shall calculate it.

Other phase-space functions can be defined and combined with other transformation rules between operators and functions in phase space, ${ }^{45}$ but the Wigner function is the only one which is in general real.

We have here defined phase-space functions for states. Equivalently, we can define phase-space functions for transitions by everywhere in Eqs. (1)-(3), (8), and (12) replacing the single state, $|\Psi\rangle$, with two different states, $\left|\Psi_{1}\right\rangle \neq\left|\Psi_{2}\right\rangle$. In the present paper we shall however focus on phase-space functions for states.

Finally, it should be mentioned that Eq. (9) offers a unique way of obtaining densities in position space for any operator and state simply by only integrating over the momentum coordinates. This has previously been demonstrated by us $^{46}$ by calculating kinetic and exchange energy densities for some molecules and atoms. The exchange energy density is particularly interesting since it does not have any classical counterpart.

In the present paper we shall solely consider the firstorder Wigner function, obtained by putting $n=1$ in the above definitions and relations. Hence, we shall omit the subscript $n$. The wave functions from which the first-order Wigner function is calculated are always chosen to be of Hartree-Fock type. This simplifies the formulas and still leads to accurate results.

In the remainder of the paper we shall use Hartree atomic units, i.e., Planck's constant, $\hbar$, the electronic mass, $m_{e}$, and the elementary charge, $e$, are all set to 1 . Thus, $h$ gets the value $2 \pi$.

\section{THE HARTREE-FOCK APPROXIMATION AND CLOSED-SHELL ATOMS}

Within the Hartree-Fock approximation the first-order density matrix takes the form

$$
\rho\left(\mathbf{r}, \mathbf{r}^{\prime}\right) \equiv \rho_{1}\left(\mathbf{r}, \mathbf{r}^{\prime}\right)=2 \sum_{i=1}^{N / 2} \phi_{i}(\mathbf{r}) \phi_{i}^{*}\left(\mathbf{r}^{\prime}\right)
$$

where $\left\{\phi_{i}\right\}$ is the set of occupied orbitals.

Wigner's first-order phase-space function is then [cf. Eq. (4)] written as a sum of single phase-space functions:

$$
\begin{aligned}
& f(\mathbf{r}, \mathbf{p}) \equiv f_{1}(\mathbf{r}, \mathbf{p})=2 \sum_{i=1}^{N / 2} f_{i}(\mathbf{r}, \mathbf{p}), \\
& f_{i}(\mathbf{r}, \mathbf{p})=\frac{1}{\pi^{3}} \int \phi_{i}\left(\mathbf{r}+\mathbf{r}^{\prime}\right) \phi_{i}^{*}\left(\mathbf{r}-\mathbf{r}^{\prime}\right) \exp \left(-2 i \mathbf{p} \cdot \mathbf{r}^{\prime}\right) d \mathbf{r}^{\prime} .
\end{aligned}
$$

The Hartree-Fock approximation is reasonable for closed-shell atoms. For those the single-electron wave functions $\phi_{i}(\mathbf{r})$ are explicitly given as

$$
\phi_{i}(\mathbf{r})=R_{n l}(r) Y_{l m}(\theta, \phi),
$$

where for the atoms we have considered, we have used the expansions of the radial dependencies in Gaussians as given by van Duijneveldt ${ }^{47}$ for helium, beryllium, and neon, and as given by Huzinaga ${ }^{48}$ for argon and zinc. For all atoms except zinc we used 10 Gaussians to describe $s$ functions and 6 to describe $p$ functions. For zinc we used 14 for $s$ functions, 9 for $p$ functions, and 5 for $d$ functions.

In order to depict the phase-space function we define in the six-dimensional phase space six new variables. As three we use the two lengths $r$ and $p$ and the angle between the two vectors, $u=\angle(\mathbf{r}, \mathbf{p})$. As the other three we use the Euler angles $\alpha, \beta, \gamma$ describing how the coordinate system $^{29}$ 


$$
\begin{aligned}
& \hat{e}_{1}=\left(\frac{\mathbf{r}}{r}-\frac{\mathrm{p}}{p}\right) /[2 \sin (u / 2)], \\
& \hat{e}_{2}=\left(\frac{\mathbf{r}}{r}+\frac{\mathrm{p}}{p}\right) /[2 \cos (u / 2)], \\
& \hat{e}_{3}=\hat{e}_{1} \times \hat{e}_{2},
\end{aligned}
$$

is orientated with respect to the reference system.

The closed-shell function (14a) can now be shown (see the Appendix) to be independent of $\alpha, \beta$, and $\gamma$, i.e., it is only a function of $r, p$, and $u$. The dependence on $u$ can be analyzed in a similar way as in Ref. 29 , but for simplicity we shall here remove the $u$ dependence by integration. Accordingly, we integrate both sides of Eq. (14a) over $u$. We also integrate over $\alpha, \beta$, and $\gamma$, which amounts to multiplying the left-hand side by $8 \pi^{2}$. Furthermore, we multiply by $r^{2} p^{2}$. We are thus left with the function

$$
F(r, p)=2 \sum_{i=1}^{N / 2} F_{i}(r, p),
$$

where

$$
\int_{0}^{\infty} \int_{0}^{\infty} F_{i}(r, p) d r d p=1
$$

As also demonstrated in the Appendix, the independence of the radial functions $R_{n l}$ of (15) of $m$ leads to the result that all functions $F_{i}(r, p)$ for same $(n, l)$ are identical. For the closed-shell atoms it is thus sufficient to calculate only one phase-space function for each $(n, l)$ shell and afterwards multiply it with the occupancy of the shell.

Finally, in the Appendix we demonstrate how the single-electron phase-space functions can be calculated and present the formulas.

\section{THE THOMAS-FERMI THEORY}

The Thomas-Fermi theory ${ }^{1-3}$ is a statistical theory for the electron densities,

$$
n(\mathbf{r})=\rho(\mathbf{r}, \mathbf{r}),
$$

in atoms and molecules. It assumes high density and small variations of it in differential parts of the threedimensional position space. In the simplest version ${ }^{1,2}$ of this theory the total energy for an atom is written as the sum of the kinetic energy of the electrons (proportional to $n^{5 / 3}$ ), the Coulomb energy from the self-interaction of the electrons, and the Coulomb energy from the interaction between the electrons and the nucleus. By requiring this energy functional of $n(\mathbf{r})$ to be stationary to small variations under the constraint that the total number of electrons is conserved, and by introducing

$$
\begin{aligned}
& x=r / v \\
& v=\frac{1}{4}\left(\frac{9 \pi^{2}}{2 Z}\right]^{1 / 3}, \\
& n(r)=\frac{1}{3 \pi^{2}}\left[\frac{2 Z \phi(r / v)}{r}\right)^{3 / 2},
\end{aligned}
$$

( $Z$ being the nuclear charge), one arrives at the following nonlinear differential equation for a neutral atom

$$
\begin{aligned}
& \phi^{\prime \prime}(x)=\phi^{3 / 2}(x) / x^{1 / 2}, \\
& \phi(0)=1, \\
& \phi\left(x_{0}\right)=\phi^{\prime}\left(x_{0}\right)=0, x_{0} \rightarrow \infty .
\end{aligned}
$$

Latter $^{49}$ gives approximate expressions for $\phi(x)$.

One of the assumptions of this theory is that for a given value of $r$ there will in the six-dimensional phase space be a constant density up to an upper limit $p_{\mu}(r)$ above which it is zero. Here,

$$
p_{\mu}(r)=[2 Z \phi(r / v) / r]^{1 / 2} .
$$

This prediction can be compared with our calculated Wigner function, which - as demonstrated in Sec. II-is to be interpreted as a quasidensity in phase space.

Due to the assumption of large electron densities, the Thomas-Fermi theory will be most accurate for $Z \rightarrow \infty .^{4,50}$

\section{FILLED $s$ SHELLS: THE HELIUM AND BERYLLIUM ATOMS}

The phase-space function for the helium atom, shown in Fig. 1(a), is simply twice that of a single $1 s$ electron. Therefore, it shows many similarities with that of the $1 s$ electron of the hydrogen atom presented in our earlier paper. ${ }^{29}$ We observe a region for small $r p$ inside which the major part of the function is located. Outside this area the Wigner function oscillates around zero with rapidly decreasing amplitude. The largest change from the phasespace function of hydrogen is the shift of the maximum to smaller $r$ and larger $p$ caused by the increased nuclear charge: For hydrogen the maximum was located at $(r, p)=(1.3,0.7)$, for helium it is found at $(0.8,1.0)$.

Except for having the same shape the Thomas-Fermi curve $p=p_{\mu}(r)$ of Fig. 1(b) shows only little agreement with the phase-space function. The Thomas-Fermi curve clearly underestimates for small $r$ values the $p$ value inside which the (major part of the) phase-space function is located. Furthermore, it does not share the property of the calculated phase-space function of being mainly located inside a region defined by a maximum $r$ value. Improvements of the Thomas-Fermi theory with the inclusion of exchange interactions (see, e.g., Ref. 3) will improve on this but only a little.

In Fig. 2(a) we depict the phase-space function of the beryllium atom. This is the sum of that of the $1 s$ shell and that of the $2 s$ shell. The former is located at even smaller $r$ values and even larger $p$ values than was the case for the hydrogen and helium atoms: a direct consequence of the increased nuclear charge.

In Fig. 3 we show the radial parts of the $1 s$ and $2 s$ wave functions in both position and momentum representation. For the $1 s$ functions we notice the small extension in the $r$ space and the larger extension in the $p$ space. For the $2 s$ functions the reverse is found. For both we notice the approximate coincidence of the positions of the extrema and the local maxima for the phase-space function. 

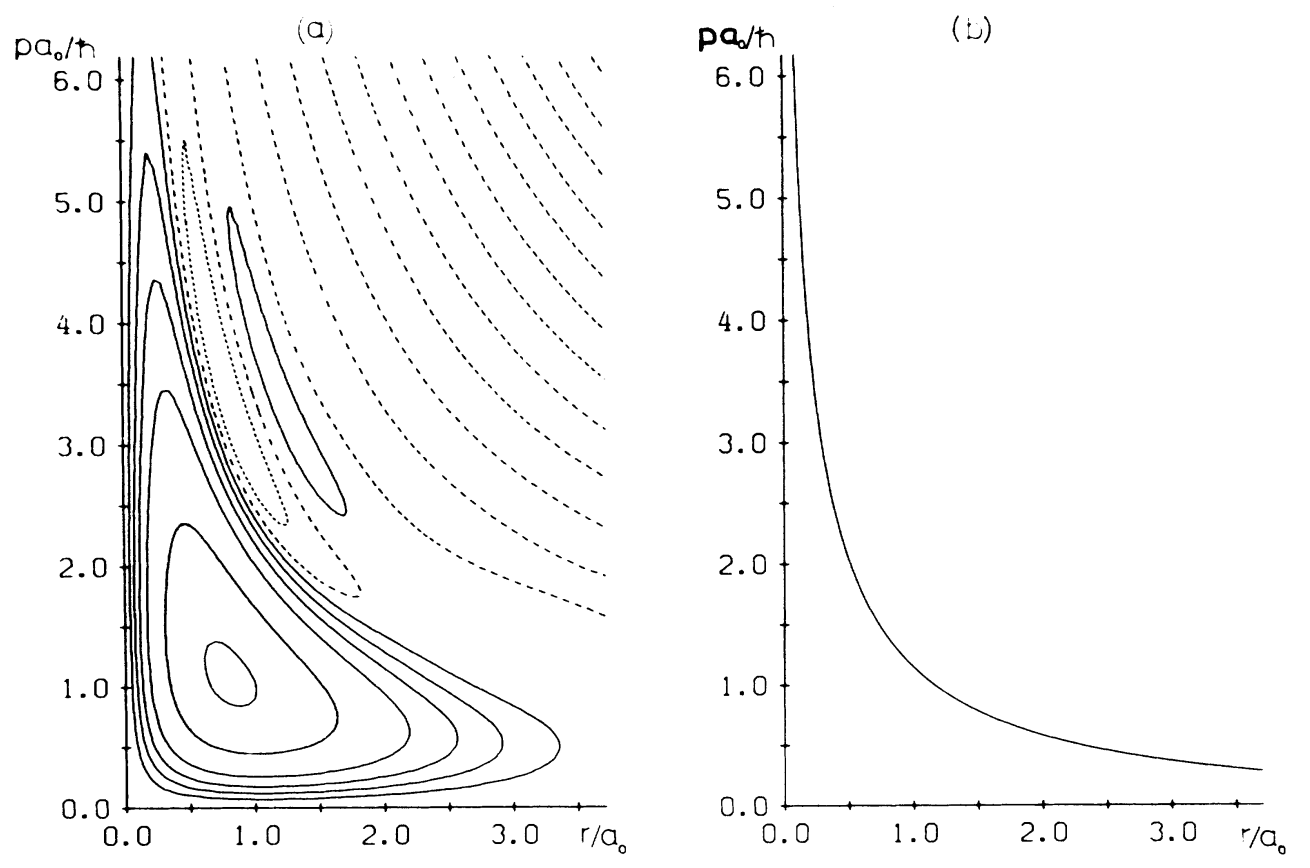

FIG. 1. (a) Contour curves for the Wigner function $F(r, p)$ for the helium atom in a $(r, p)$ phase plane. The contour values are the following: positive (solid curves), 1.0, 0.5, 0.2, 0.1, 0.05, 0.02; dashed, 0.0; negative (dotted), -0.02 . (b) The Thomas-Fermi curve $p=p_{\mu}(r)$ for the helium atom.
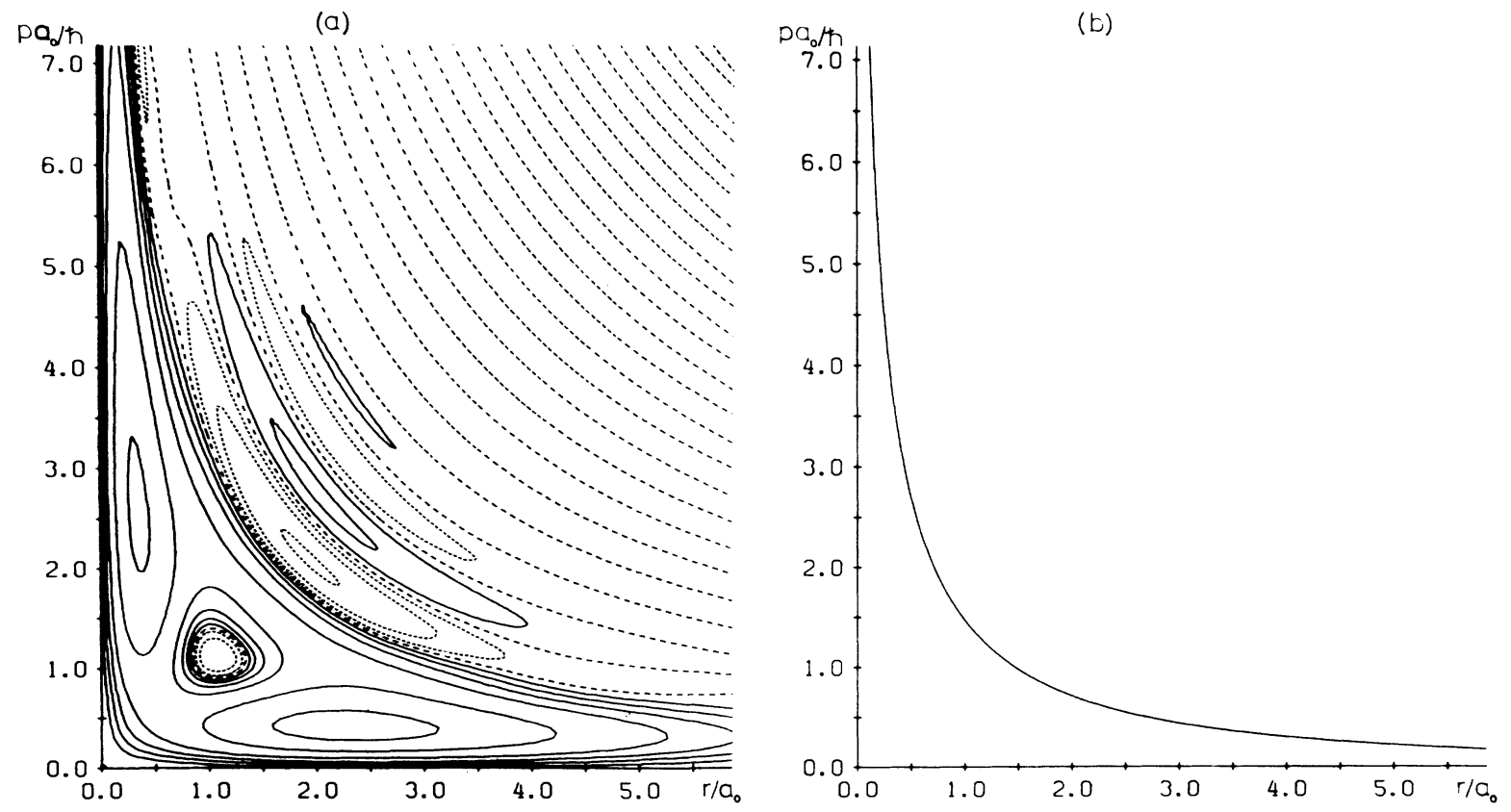

FIG. 2. As for Fig. 1 but for the beryllium atom. In the contour values the two negative values (dotted curves) -0.05 and -0.1 have been included. 

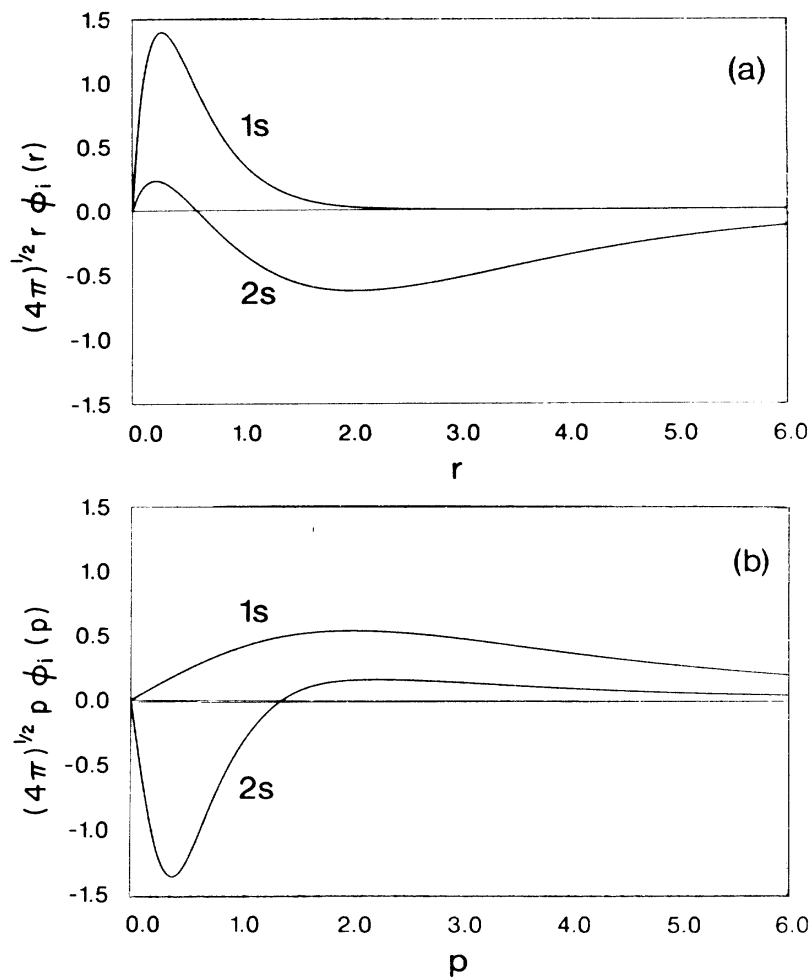

FIG. 3. The radial dependencies of the wave functions for the $1 s$ and $2 s$ electrons of beryllium in position (a) and momentum (b) representation. The functions are multiplied by $(4 \pi)^{1 / 2} r$ (a) or $(4 \pi)^{1 / 2} p(\mathrm{~b})$.

The local minimum of the phase-space function around $(r, p)=(1.2,1.2)$ is related to the nodes of the radial parts of the wave functions. The one-dimensional analogue of Eq. (14b) is for real wave functions

$f_{i}(r, p)=\frac{2}{\pi} \int_{0}^{\infty} \phi_{i}\left(r+r^{\prime}\right) \phi_{i}\left(r-r^{\prime}\right) \cos \left(2 p r^{\prime}\right) d r^{\prime}$.
We see that in this case the phase-space function is the self-convolution of the wave function modified by a cosine. A similar expression is valid for the (real) wave functions in momentum space. Both curves for the $2 s$ wave functions in Fig. 3 have two extrema, a maximum and a minimum, and are fairly localized. Therefore, when $r$, respectively $p$, is roughly in the middle between the two extrema, the phase-space function will be most negative. The cosine factor is positive in the region where the integrand has its largest support. These argumentscarried through in the one-dimensional space-will be slightly modified in the three-dimensional space.

In Fig. 2(a) it is interesting to notice that the volumes of the two regions of the two different shells are comparable. Thus, a small extension in position space leads to a large extension in momentum space, and vice versa; this is just another way of stating Heisenberg's uncertainty relation.

Finally, the agreement between the Thomas-Fermi curve $p=p_{\mu}(r)$ shown in Fig. 2(b) and the phase-space function is improved as compared with the helium atom, although the disagreements noticed for the helium atom remain.

\section{FILLED $s$ AND $p$ SHELLS: THE NEON AND ARGON ATOMS}

For the neon atom we depict in Fig. 4 the contributions to the total phase-space function from the $1 s$ shell [Fig. 4(a)], the $2 s$ shell [Fig. 4(b)], and the $2 p$ shell [Fig. 4(c)]. The shift towards smaller $r$ and larger $p$ values for the $1 s$ and $2 s$ shells as compared with beryllium is clearly seen. Furthermore, we recognize in Fig. 4(b) both the positive and negative parts of the phase-space function discussed in the previous section for the beryllium $2 s$ shell.

Comparing Figs. 4(a) and 4(b) we see that the oscillations for larger $r p$ have larger amplitudes for the $2 s$ shell than for the $1 s$ shell. For the $2 p$ shell these become even larger but, on the other hand, the $2 p$ shell contains six compared with two electrons of the $s$ shells.

We rewrite the phase-space function as

$$
\left.f_{i}(\mathbf{r}, \mathbf{p})=\frac{1}{\pi^{3}} \iint \phi_{i}\left(\mathbf{r}+\mathbf{r}^{\prime}\right) \phi_{i}^{*}\left(\mathbf{r}-\mathbf{r}^{\prime}\right) d \mathbf{r}^{\prime}+\int \phi_{i}\left(\mathbf{r}+\mathbf{r}^{\prime}\right) \phi_{i}^{*}\left(\mathbf{r}-\mathbf{r}^{\prime}\right)\left[\exp \left(-2 i \mathbf{p} \cdot \mathbf{r}^{\prime}\right)-1\right] d \mathbf{r}^{\prime}\right]
$$

For small $p$ the first part is dominating, and the phasespace function is essentially the self-convolution of the wave function. A similar argumentation can be applied for $r$ small by considering the wave function in the momentum representation. Due to the inversion antisymmetry about the origin for the wave function in either position or momentum representation of the $2 p$ orbitals, the phase-space function is negative for small $r p$ values as seen in Fig. 4(c).

The total phase-space function presented in Fig. 4(d) shows less details than the single ones for each shell: It mainly shows similarity with that of the $2 p$ shell whereas the detailed structures of the $1 s$ and the $2 s$ shells are smeared out. This is of course partly a consequence of the different occupancy of the shells. For the beryllium atom it was still possible to identify the contribution of each shell. But because of the roughly constant radius in position space for all atoms the single-shell contributions get smeared out by those of the other shells: a statistical theory becomes more justified. The improved agreement with the theory of Thomas and Fermi can be seen by comparing the Thomas-Fermi curve $p=p_{\mu}(r)$ shown in Fig. 4(e) with the phase-space function.

For argon we show in Fig. 5(a) the total phase-space function. Although the relative number of $p$ electrons has increased compared with that of neon (12 out of $18 \mathrm{com}$ - 
(a)

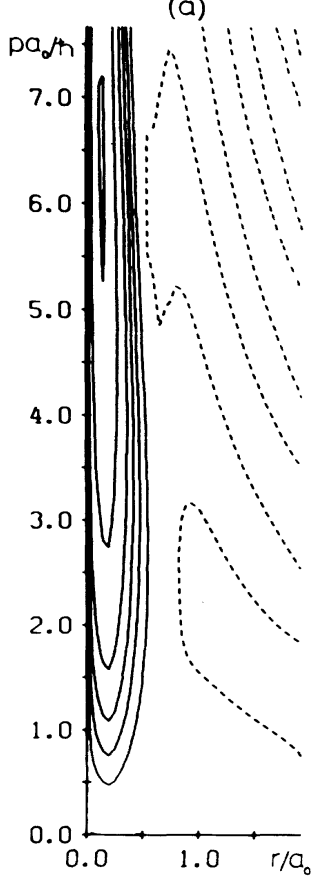

(b)

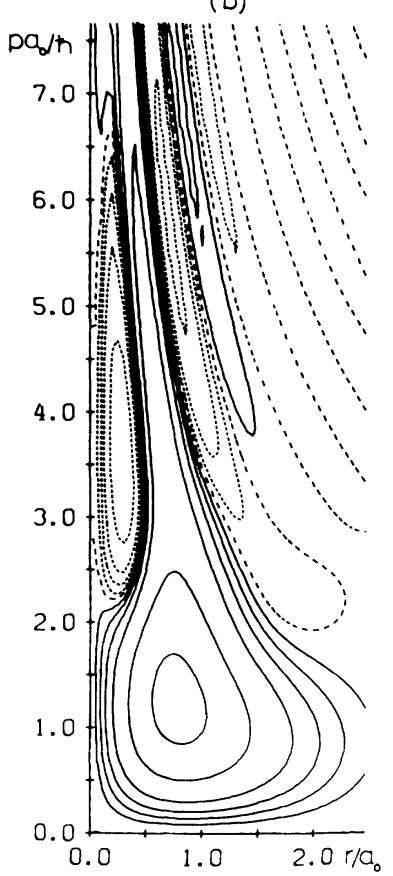

(c)

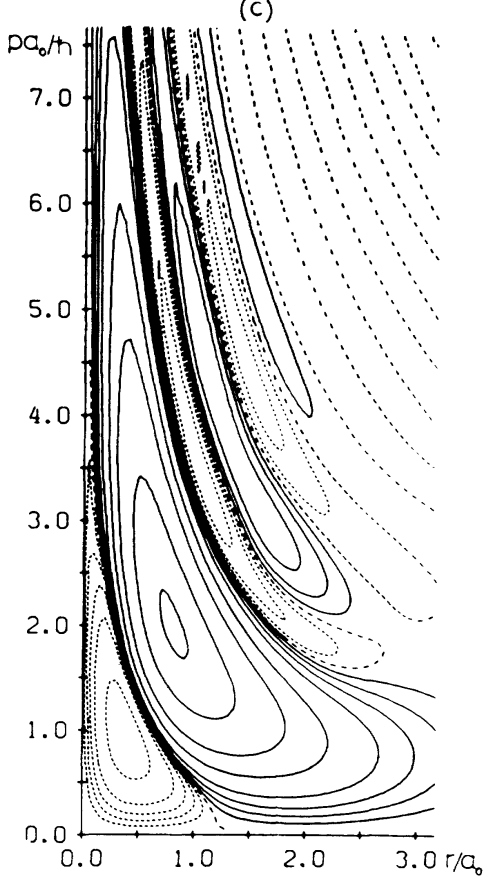

(d)

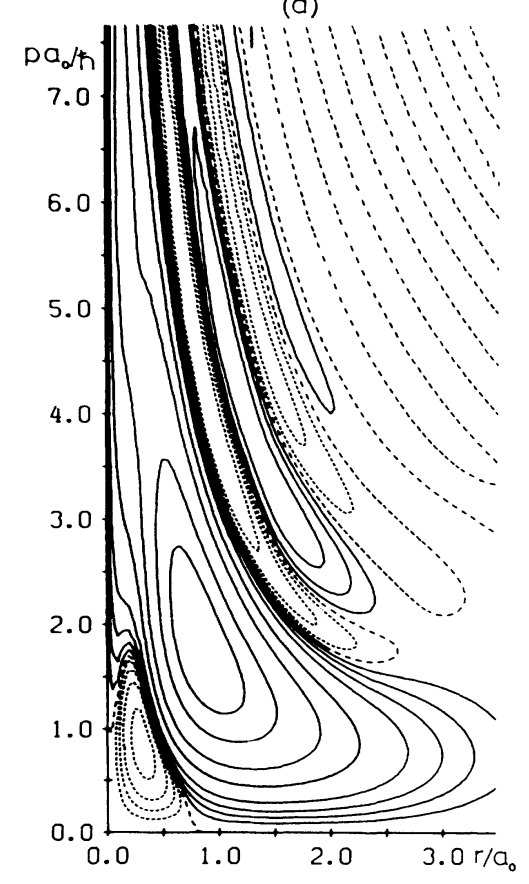

(e)

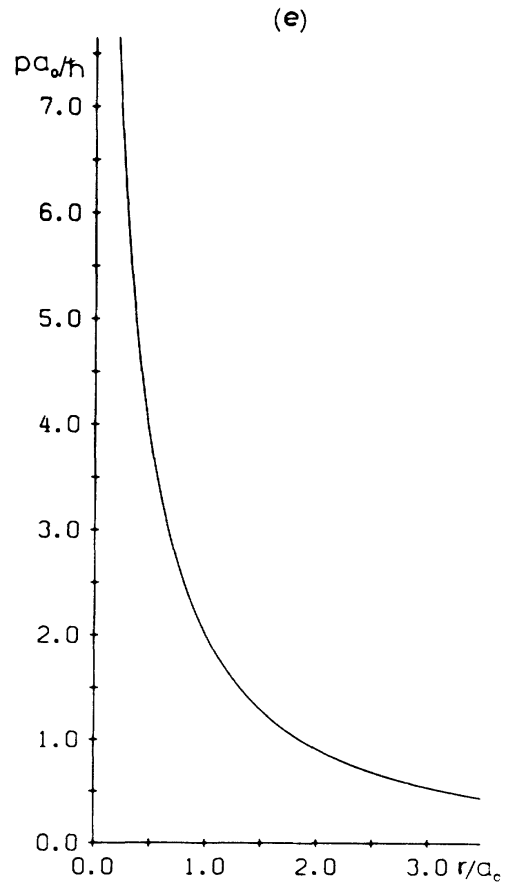

FIG. 4. Contour curves for (a) the $1 s$ contribution, (b) the $2 s$ contribution, and (c) the $2 p$ contribution to (d) the total Wigner function $F(r, p)$ for the neon atom in a $(r, p)$ phase plane. The contour values are the following: positive (solid curves), 3.0, 2.0, 1.0, 0.5, 0.2, $0.1,0.05,0.02$; dashed, 0.0 ; negative (dashed curves), $-0.02,-0.05,-0.1,-0.2,-0.5$. (e) The Thomas-Fermi curve $p=p_{\mu}(r)$ for the neon atom. 

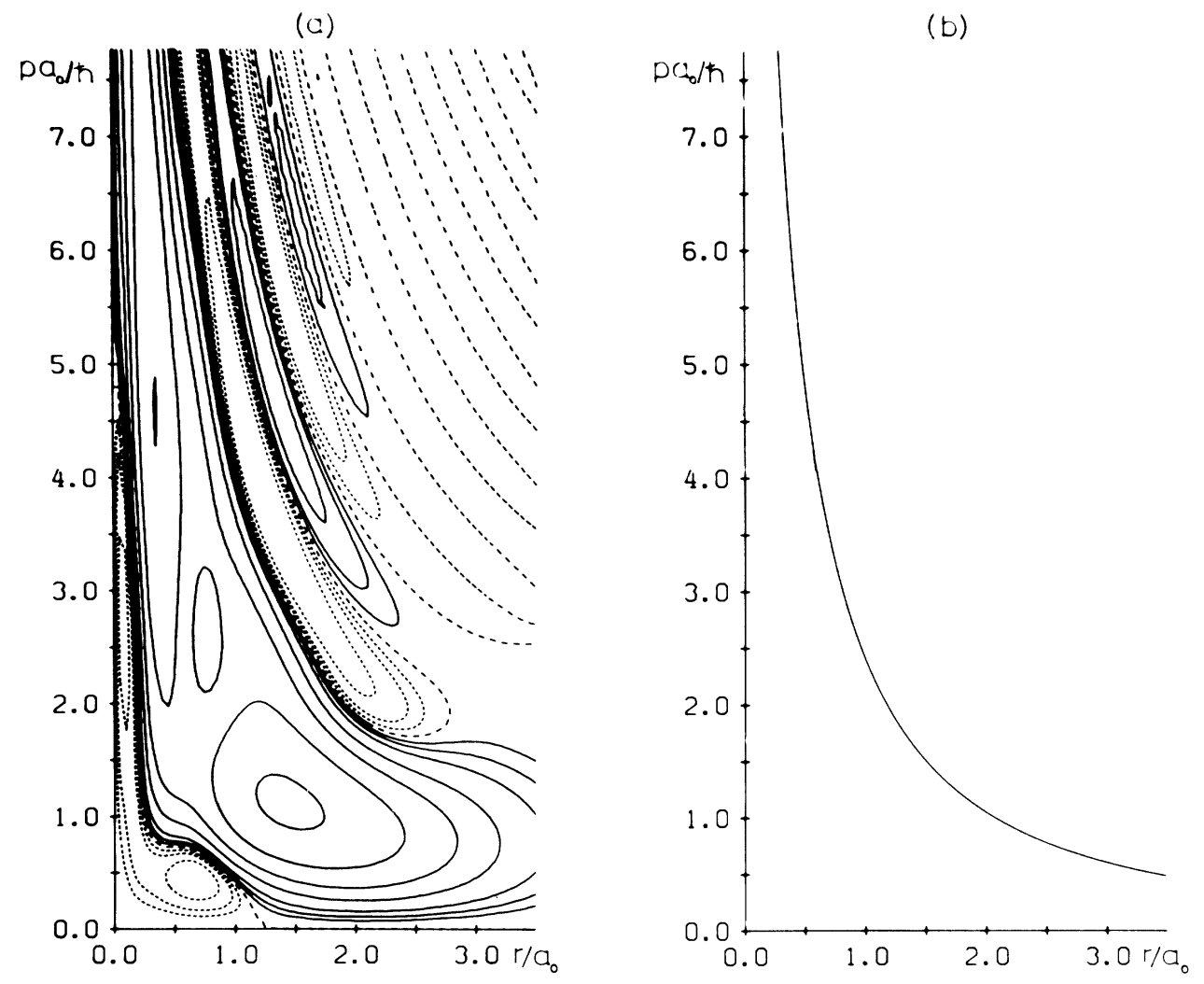

FIG. 5. (a) Contour curves for the Wigner function $F(r, p)$ for the argon atom in a $(r, p)$ phase plane. The contour values are the following: positive (solid curves), 4.0, 2.0, 1.0, 0.5, 0.2, 0.1, 0.05; dashed, 0.0; negative (dotted curves), $-0.05,-0.1,-0.2$. (b) The Thomas-Fermi curve $p=p_{\mu}(r)$ for the argon atom.

pared with 6 out of 10), the volume of that part of phase space for small $r p$ where the total function is negative has decreased. Just outside this region we recognize a fairly large region where the Wigner function is large and only shows few details: the statistical description becomes still better. The improved agreement with the Thomas-Fermi curve of Fig. 5(b) confirms this.

\section{FILLED $s, p$, AND $d$ SHELLS: THE ZINC ATOM}

The contribution of the $3 d$ shell to the total Wigner function for the zinc atom is depicted in Fig. 6(a). The angular parts of the wave function in position or momentum representation have two nodal planes. These show up in the phase-space representation as the two nodal curves of the phase-space function for small $r p$. A combined argumentation of those carried through for explaining the regions of negative phase-space function for the beryllium $2 s$ electrons and the neon $2 p$ electrons will show this.

Although many of the shells for the zinc atom have regions of negative phase-space function for smaller $r p$ the superposition of those leads to a total phase-space function which only has a smaller region for small $r p$ where it is negative, as seen in Fig. 6(b). In this figure we clearly see that the oscillations around zero further away from the origin have by far the largest amplitude for (small $r$, large $p$ ) compared with the amplitude of the oscillations for (large $r$, small $p$ ). The agreement with the ThomasFermi curve $p=p_{\mu}(r)$ in Fig. 6(c) is reasonable but no more.

Since zinc is the largest atom in the present analysis, we shall make a more detailed comparison with the ThomasFermi theory. If the Wigner function $F(r, p)$ had agreed with the electron density in phase space as obtained in the Thomas-Fermi theory, the contour curves for $F(r, p)$ would have been contour curves for $r^{2} p^{2}$ [i.e., hyperbolae in a $(r, p)$ plane] below the curve $p=p_{\mu}(r)$ (cf. Sec. IV). These are shown in Fig. 7 for the zinc atom. We see that the $p_{\mu}$ curve falls off faster than any hyperbola (otherwise it would not have been normalizable) such that more density is accumulated for (small $r$, large $p$ ) than for (large $r$, small $p$ ). This is actually in agreement with the behavior of the calculated phase-space function. But the ThomasFermi theory predicts the largest densities for $(r \rightarrow 0, p \rightarrow \infty)$. This is in contrast with the (nonzero $r$, finite $p$ ) positions of the maxima of $F(r, p)$ for all atoms of finite nuclear charge. The existence of a maximum $p$ value for any value of $r, p=p_{\mu}(r)$, as predicted by the simplest Thomas-Fermi theory or later improvements, is not in 

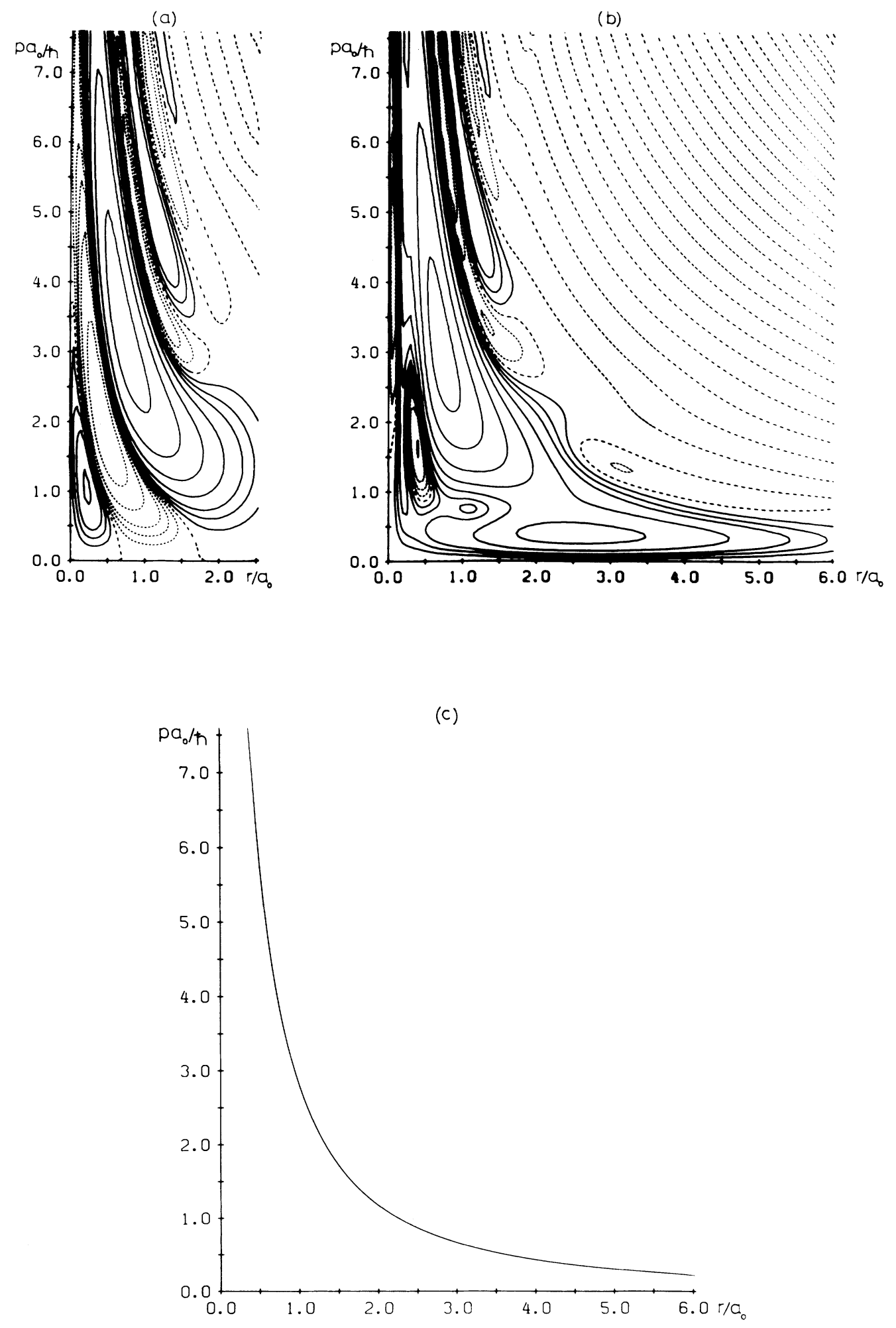

FIG. 6. (a) Contour curves for the $3 d$ contribution to (b) the total Wigner function $F(r, p)$ for the zinc atom in a $(r, p)$ phase plane. The contour values are the following: positive (solid curves), 7.0, 4.0, 2.0, 1.0, 0.5, 0.25, 0.15, 0.07; dashed, 0.0; negative (dotted curves), $-0.07,-0.15,-0.25,-0.5,-1.0$. (c) The Thomas-Fermi curve $p=p_{\mu}(r)$ for the zinc atom. 


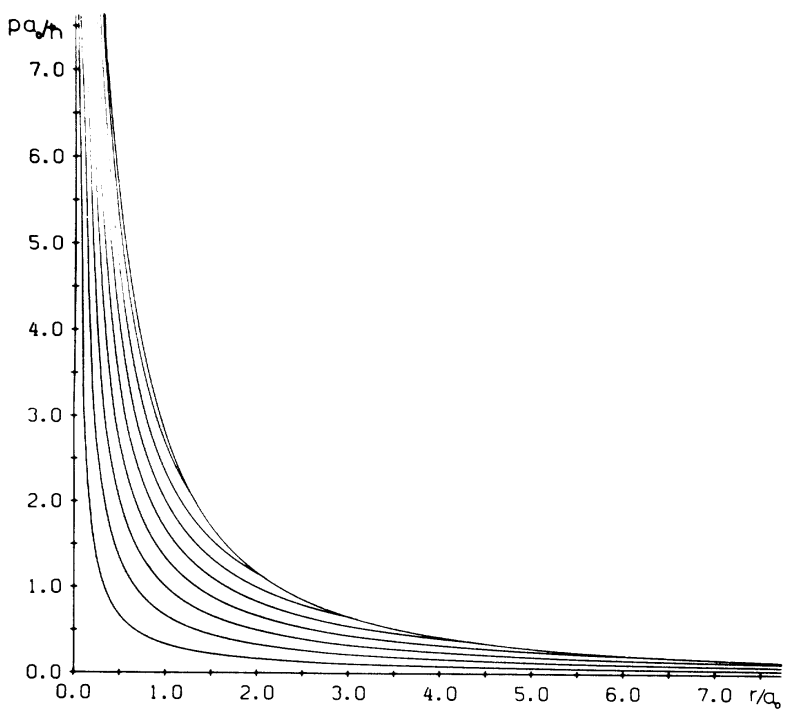

FIG. 7. Contour curves for $r p$ in a $(r, p)$ phase plane inside the Thomas-Fermi curve $p=p_{\mu}(r)$ for the zinc atom. Contour values: $0.3,0.6,0.9,1.2,1.5,1.8,2.1,2.4$.

agreement with the phase-space function results especially for small $p$.

\section{CONCLUSION}

In the present paper, as well as in our earlier ones on phase-space functions of electronic ground states of atomic and molecular systems, ${ }^{29,33}$ we have shown that the phase-space function offers a useful alternative of presenting and analyzing the electronic state.

The phase-space function allows a simultaneous description of position and momentum coordinates. As a result we have for the closed-shell atoms, analyzed in the present paper, seen how the $1 s$ shell moves towards smaller $r$ and larger $p$ values with increasing atomic number. As a consequence of Heisenberg's uncertainty relations the contraction in $\mathbf{r}$ space is followed by an elongation in p space. The wave functions in coordinate or momentum representation of the states of the other shells have nodes in the radial and/or angular dependencies. We have here demonstrated how these nodes are refound in the phase space as regions of negative phase-space function.

The results of the statistical theory of Thomas and Fermi or later improvements is in partial agreement with the Wigner function, but many of the details of the latter are lacking in the former, especially the description of the behavior for large $r p$.

The Hartree-Fock approximation used here allowed a simple separation into orbital phase-space functions. For systems where this approximation is too crude, the natural orbitals of Löwdin ${ }^{36}$ can be used in a similar separation.

The Wigner phase-space function contains no more and no less information than the wave functions (in contrast with for instance the Husimi function, ${ }^{35,51}$ which is obtained by folding the Wigner function with Gaussians giving an everywhere non-negative function, but for which information is lost due to the folding). But the Wigner function makes a more direct comparison with classical descriptions possible such that quantum effects easily are recognized. This was in detail demonstrated for the hydrogen atom. ${ }^{29}$ Furthermore, detailed information on the effects of the formation of a chemical bond can easily be extracted as shown for the $\mathrm{LiH}$ molecule. ${ }^{31}$ The present paper is hoped to demonstrate the usefulness of the Wigner phase-space function.

\section{ACKNOWLEDGMENTS}

We are grateful to Professor A. J. Thakkar and Professor W. Weyrich for valuable discussions. Furthermore, we want to thank Dr. H. Johansen for letting us use his density contour programs.

\section{APPENDIX}

In this appendix we shall investigate general phasespace functions for atomic orbitals, thereby proving that the single-orbital phase-space function, $F_{i}(r, p)$ of Sec. III, for given $(n, l)$ is independent of $m$ and that the closed-shell function (14a) is independent of $\alpha, \beta$, and $\gamma$. Furthermore, we demonstrate how $f_{i}(\mathbf{r}, \mathbf{p})$ and $F_{i}(r, p)$ can be calculated and give the final formulas when the radial dependences of the wave functions in the coordinate representation are expanded in Gaussians.

We consider the general single-particle phase-space function in the six-dimensional phase space

$$
f_{a b}(\mathbf{r}, \mathbf{p})=\frac{1}{\pi^{3}} \int \psi_{b}^{*}\left(\mathbf{r}-\mathbf{r}^{\prime}\right) \psi_{a}\left(\mathbf{r}+\mathbf{r}^{\prime}\right) \exp \left(-2 i \mathbf{p} \cdot \mathbf{r}^{\prime}\right) d \mathbf{r}^{\prime}
$$

where, for $\Psi_{a}=\Psi_{b}$, it is the phase-space function of a state; otherwise that of a transition. The wave functions are atomic orbitals, i.e.,

$$
\begin{aligned}
\psi(\mathbf{r}) & =R_{n l}(r) Y_{l m}(\theta, \phi) \\
& =R_{n l}(r)(-1)^{l} Y_{l m}(\pi-\theta, \phi+\pi) .
\end{aligned}
$$

Then (A1) can be written as

$$
\begin{aligned}
f_{a b}(\mathbf{r}, \mathbf{p})=\frac{1}{\pi^{3}}(-1)^{l_{b}} \int & \psi_{b}^{*}\left(\mathbf{r}^{\prime}-\mathbf{r}\right) \psi_{a}\left(\mathbf{r}^{\prime}+\mathbf{r}\right) \\
& \times \exp \left(-2 i \mathbf{p} \cdot \mathbf{r}^{\prime}\right) d \mathbf{r}^{\prime} .
\end{aligned}
$$

The integral (A3) is, for a fixed value of $\mathbf{r}$, the Fourier transform of the product of two orbitals $\psi_{a}$ and $\psi_{b}$, centered around the points $-\mathbf{r}$ and $\mathbf{r}$, respectively. Thus, it is recognized as a generalized scattering factor. The various methods for the evaluation of such factors are reviewed in Ref. 29.

Let us now place local coordinate systems at $-\mathbf{r}$ and $\mathbf{r}$, with axes which are parallel to the axes $\widehat{e}_{1}, \widehat{e}_{2}, \widehat{e}_{3}$, as defined by Eq. (16). We shall then denote the spherical polar coordinates of $\mathbf{r}^{\prime}+\mathbf{r}$ by $\left(r_{a}, \theta_{a}, \phi_{a}\right)$ and of $\mathbf{r}^{\prime}-\mathbf{r}$ by $\left(r_{b}, \theta_{b}, \phi_{b}\right)$, when referred to these local "inner" coordinate systems. Similarly, we place local coordinate systems with axes which are parallel to the axes of the outer coordinate system in which the functions (A2) are defined. With respect to these "outer" axes, $\mathbf{r}^{\prime}+\mathbf{r}$ and $\mathbf{r}^{\prime}-\mathbf{r}$ have the spherical polar coordinates $\left(r_{a}, \widetilde{\theta}_{a}, \widetilde{\phi}_{a}\right)$ and $\left(r_{b}, \widetilde{\theta}_{b}, \widetilde{\phi}_{b}\right)$, 
respectively. We may thus write

$$
\begin{aligned}
\psi_{b}^{*}\left(\mathbf{r}^{\prime}-\mathbf{r}\right) \psi_{a} & \left(\mathbf{r}^{\prime}+\mathbf{r}\right) \\
= & R_{n_{b} l_{b}}\left(r_{b}\right) R_{n_{a} l_{a}}\left(r_{a}\right) Y_{l_{b} m_{b}}^{*}\left(\widetilde{\theta}_{b}, \widetilde{\phi}_{b}\right) Y_{l_{a} m_{a}}\left(\widetilde{\theta}_{a}, \widetilde{\phi}_{a}\right) .
\end{aligned}
$$

The three Euler angles $(\alpha, \beta, \gamma) \equiv \Omega$ are defined to describe how the inner axes are rotated with respect to the outer axes, and the set $\Omega^{-1}$ is defined to describe how the outer axes are rotated with respect to the inner ones.

$$
\begin{aligned}
Y_{l_{b} m_{b}}^{*}\left(\widetilde{\theta}_{b}, \widetilde{\phi}_{b}\right) Y_{l_{a} m_{a}}\left(\widetilde{\theta}_{a}, \widetilde{\phi}_{a}\right) & =\sum_{m_{b}^{\prime} m_{a}^{\prime}} Y_{l_{b} m_{b}^{\prime}}^{*}\left(\theta_{b}, \phi_{b}\right) Y_{l_{a} m_{a}^{\prime}}\left(\theta_{a}, \phi_{a}\right)\left[D_{m_{b}^{\prime} m_{b}}^{l_{b}}\left(\Omega^{-1}\right)\right]^{*} D_{m_{a}^{\prime} m_{a}}^{l_{a}}\left(\Omega^{-1}\right) \\
& =\sum_{m_{b}^{\prime} m_{a}^{\prime}} Y_{l_{b} m_{b}^{\prime}}^{*}\left(\theta_{b}, \phi_{b}\right) Y_{l_{a} m_{a}^{\prime}}\left(\theta_{a}, \phi_{a}\right) D_{m_{b} m_{b}^{\prime}}^{l_{b}}(\Omega)\left[D_{m_{a} m_{a}^{\prime}}^{l_{a}}(\Omega)\right]^{*},
\end{aligned}
$$

where we have used the unitarity of the $D$ matrices. Thus, by writing (A3) as

$$
f_{a b}(\mathbf{r}, \mathbf{p})=(-1)^{l_{b}} \sum_{m_{b}^{\prime} m_{a}^{\prime}} D_{m_{b} m_{b}^{\prime}}^{l_{b}}(\Omega)\left[D_{m_{a} m_{a}^{\prime}}^{l_{a}}(\Omega)\right]^{*} \frac{1}{\pi^{3}} \int \psi_{n_{b} l_{b} m_{b}^{\prime}}^{*}\left(r_{b}, \theta_{b}, \phi_{b}\right) \psi_{n_{a} l_{a} m_{a}^{\prime}}\left(r_{a}, \theta_{a}, \phi_{a}\right) \exp \left(-2 i \mathbf{p} \cdot \mathbf{r}^{\prime}\right) d \mathbf{r}^{\prime},
$$

the dependence of $f_{a b}$ on the orientation of the inner axes (or, equivalently, of $\mathbf{r}$ and $\mathbf{p}$ ) with respect to the outer axes is completely put into the $D$ matrices.

We can then apply the orthogonality relations ${ }^{52}$

$$
\int D_{m \mu}^{l}(\Omega)\left[D_{m^{\prime} \mu^{\prime}}^{l^{\prime}}(\Omega)\right]^{*} d \Omega=\frac{8 \pi^{2}}{2 l+1} \delta_{l l^{\prime}} \delta_{m m^{\prime}} \delta_{\mu \mu^{\prime}}
$$

to give, for $n_{a}=n_{b}=n, m_{a}=m_{b}=m, l_{a}=l_{b}=l$ :

$$
\int f_{a b}(\mathbf{r}, \mathbf{p}) d \Omega=\frac{8 \pi^{2}}{2 l+1}(-1)^{l} \sum_{m} \frac{1}{\pi^{3}} \int \psi_{n l m}^{*}\left(r_{b}, \theta_{b}, \phi_{b}\right) \psi_{n l m}\left(r_{a}, \theta_{a}, \phi_{a}\right) \exp \left(-2 i \mathbf{p} \cdot \mathbf{r}^{\prime}\right) d \mathbf{r}^{\prime},
$$

which is independent of $m$. After integrating over the angle $u$, it is of course still independent of $m$. This is the important result stated in Sec. III and used in the calculations of $F(r, p)$.

By using the relation ${ }^{52}$

$$
\sum_{m} D_{m \mu}^{l}(\Omega)\left[D_{m \mu^{\prime}}^{l}(\Omega)\right]^{*}=\delta_{\mu \mu^{\prime}}
$$

for $n_{a}=n_{b}=n, m_{a}=m_{b}=m, l_{a}=l_{b}=l$, we also get, from Eq. (A6):

$$
\sum_{m=-l}^{l} f_{a b}(\mathbf{r}, \mathbf{p})=(-1)^{l} \sum_{m} \frac{1}{\pi^{3}} \int \psi_{n l m}^{*}\left(r_{b}, \theta_{b}, \phi_{b}\right) \psi_{n l m}\left(r_{a}, \theta_{a}, \phi_{a}\right) \exp \left(-2 i \mathbf{p} \cdot \mathbf{r}^{\prime}\right) d \mathbf{r}^{\prime} .
$$

This result shows that the closed-shell function (14a) is independent of the Euler angles $\alpha, \beta, \gamma$, as stated in Sec. III.

We will now restrict ourselves to the case that the radial dependence of the wave function in position space, $R_{n l}$ of Eq. (A2), is given as a sum of Gaussians, i.e.

$$
R_{n l}=\sum_{i} c_{i} N\left(l ; \alpha_{i}\right) r^{l} \exp \left(-\alpha_{i} r^{2}\right)
$$

where the normalization constants are

$$
N(l ; \alpha)=\left(\frac{2(4 \alpha)^{n+1}}{(2 n+1) ! !}(2 \alpha / \pi)^{1 / 2}\right)^{1 / 2}=\left[\frac{4 \alpha^{n}}{(2 n+1) ! !}\right)^{1 / 2} \times N(0 ; \alpha) .
$$

The phase-space function (A1) is then

$f_{a b}(\mathbf{r}, \mathbf{p})=\frac{1}{\pi^{3}} \sum_{j k} c_{j} c_{k}^{*} N\left(l_{a} ; \alpha_{j}\right) N\left(l_{b} ; \alpha_{k}\right) \int r_{a}^{l_{a}} r_{b}^{l_{b}} \exp \left(-\alpha_{j} r_{a}^{2}\right) \exp \left(-\alpha_{k} r_{b}^{2}\right) Y_{l_{a} m_{a}}\left(\widetilde{\theta}_{a}, \widetilde{\phi}_{a}\right) Y_{l_{b} m_{b}}^{*}\left(\widetilde{\theta}_{b}, \widetilde{\phi}_{b}\right) \exp \left(-2 i \mathbf{p} \cdot \mathbf{r}^{\prime}\right) d \mathbf{r}^{\prime}$.

The function $r^{l} Y_{l m}(\widetilde{\theta}, \widetilde{\phi})$ is an $l$ th order homogeneous polynomial in the Cartesian coordinates $x, y, z$. This polynomial is denoted $P_{l m}(x, y, z)$. Then we can write (A13) as a linear combination of terms of the type

$$
\begin{aligned}
g_{a b}(\mathbf{r}, \mathbf{p})=\frac{1}{\pi^{3}} N\left(l_{a} ; \alpha\right) N\left(l_{b} ; \beta\right) \int & P_{l_{a} m_{a}}\left(x-x^{\prime}, y-y^{\prime}, z-z^{\prime}\right) P_{l_{b} m_{b}}^{*}\left(x+x^{\prime}, y+y^{\prime}, z+z^{\prime}\right) \\
& \times \exp \left(-\alpha\left|\mathbf{r}-\mathbf{r}^{\prime}\right|^{2}\right) \exp \left(-\beta\left|\mathbf{r}+\mathbf{r}^{\prime}\right|^{2}\right) \exp \left(-2 i \mathbf{p} \cdot \mathbf{r}^{\prime}\right) d \mathbf{r}^{\prime} .
\end{aligned}
$$


It is now easily realized that the polynomials $P_{l m}$ can be put outside the integration, when replacing it with operators:

$$
P_{l m}\left(x \pm x^{\prime}, y \pm y^{\prime}, z \pm z^{\prime}\right) \rightarrow P_{l m}\left\{x \pm \frac{i}{2} \frac{\partial}{\partial p_{x}}, y \pm \frac{i}{2} \frac{\partial}{\partial p_{y}}, z \pm \frac{i}{2} \frac{\partial}{\partial p_{z}}\right\}
$$

such that from the integrals for $l_{a}=l_{b}=m_{a}=m_{b}=0$ we can derive all the others by simple multiplications and differentiations.

For $l_{a}=l_{b}=m_{a}=m_{b}=0$ we have

$$
g_{a b}(\mathbf{r}, \mathbf{p})=\frac{1}{\pi^{3}}(\gamma / \sigma)^{3 / 4} \exp \left(-\gamma r^{2}-p^{2} / \sigma\right) \cos (2 \tau \mathbf{r} \cdot \mathbf{p}),
$$

where

$$
\begin{aligned}
& \gamma=\frac{4 \alpha \beta}{\alpha+\beta}, \\
& \sigma=\alpha+\beta, \\
& \tau=\frac{\alpha-\beta}{\alpha+\beta} .
\end{aligned}
$$

In calculating the phase-space function $F_{i}(r, p)$, only depending on the lengths of the vectors $\mathbf{r}$ and $\mathbf{p}$, for $n_{a}=n_{b}=n$, $l_{a}=l_{b}=l$, and $m_{a}=m_{b}=m$, we can make use of the independency of this function of $m$ and thus calculate it for a simple case for which $P_{l m}$ reduces to a single term.

Explicitly we find

$$
F_{n l m}(r, p)=\sum_{j k} c_{j} c_{k}^{*} G_{l}\left(\alpha_{j}, \alpha_{k} ; r, p\right)
$$

where the expression for $G$ for $l=0,1,2$ is for $l=0$,

$$
G_{0}(\alpha, \beta ; r, p)=\frac{16}{\pi} r^{2} p^{2}(\gamma / \sigma)^{3 / 4} \exp \left(-\gamma r^{2}\right) \exp \left(-p^{2} / \sigma\right) j_{0}(2 \tau r p),
$$

for $l=1$,

$$
G_{1}(\alpha, \beta ; r, p)=\frac{32}{3 \pi}(\gamma / \sigma)^{5 / 4} \exp \left(-\gamma r^{2}\right) \exp \left(-p^{2} / \sigma\right) \times\left\{j_{0}(2 \tau r p)\left[\gamma r^{2}+p^{2} / \sigma-\frac{1}{2}\right]-\cos (2 \tau r p)\right\},
$$

and for $l=2$,

$$
\begin{aligned}
G_{2}(\alpha, \beta ; r, p)= & \frac{16}{15 \pi}(\gamma / \sigma)^{7 / 4} \exp \left(-\gamma r^{2}\right) \exp \left(-p^{2} / \sigma\right) \\
\times & \left\{j_{0}(2 \tau r p)\left[4 \gamma^{2} r^{4}+4 p^{4} / \sigma^{2}+8 \gamma r^{2} p^{2} / \sigma-16 \tau^{2} r^{2} p^{2}-12 \gamma r^{2}-12 p^{2} / \sigma+7+4 \gamma / \sigma / \tau^{2}\right]\right. \\
& \left.\quad+\cos (2 \tau r p)\left[-8 \gamma r^{2}-8 p^{2} / \sigma+8-4 \gamma / \sigma / \tau^{2}\right]\right\}
\end{aligned}
$$

where $j_{0}(x)$ is the spherical Bessel function of zeroth order:

$$
j_{0}(x)=\frac{\sin (x)}{x} \text {. }
$$

*Present address.

${ }^{1}$ L. H. Thomas, Proc. Cambridge Philos. Soc. 23, 542 (1926).

${ }^{2}$ E. Fermi, Z. Phys. 48, 73 (1928).

${ }^{3} \mathrm{P}$. Gombás, Die Statistische Theorie des Atoms und ihre Anwendungen (Springer-Verlag, Berlin, 1949).

${ }^{4}$ N. H. March, in Theory of the Inhomogeneous Electron Gas, edited by S. Lundqvist and N. H. March (Plenum Press, New York, 1983).

${ }^{5}$ E. Wigner, Phys. Rev. 40, 749 (1932).

${ }^{6} \mathrm{H}$. Weyl, The Theory of Groups and Quantum Mechanics (Dover, New York, 1931).
${ }^{7}$ H. Groenewold, Physica 12, 405 (1946).

${ }^{8}$ E. J. Moyal, Proc. Cambridge Philos. Soc. 45, 99 (1949).

${ }^{9}$ S. R. de Groot and L. G. Suttorp, Foundations of Electrodynamics (North-Holland, Amsterdam, 1972).

${ }^{10} \mathrm{R}$. Balescu, Equilibrium and Nonequilibrium Statistical Mechanics (John Wiley and Sons, New York, 1975).

${ }^{11}$ W. H. Louisell, Quantum Statistical Properties of Radiation (John Wiley and Sons, New York, 1973).

${ }^{12}$ W. R. Frensley, Phys. Rev. Lett. 57, 2853 (1986).

${ }^{13}$ M. J. Bastiaans, Opt. Acta 26, 1333 (1979).

${ }^{14}$ R. C. Brown and E. J. Heller, J. Chem. Phys. 75, 186 (1981). 
15J. S. Hutchinson and R. E Wyatt, Phys. Rev. A 23, 1567 (1981).

${ }^{16}$ H. Esbensen, A. Winther, R. A. Broglia, and C. H. Dasso, Phys. Rev. Lett. 41, 296 (1978).

${ }^{17}$ N. E. Henriksen, Chem. Phys. Lett. 121, 139 (1985).

${ }^{18}$ N. L. Balazs and B. K. Jennings, Phys. Rep. 104, 347 (1984).

${ }^{19}$ M. Hillery, R. F. O'Connell, M. O. Scully, and E. P. Wigner, Phys. Rep. 106, 121 (1984).

${ }^{20}$ M. V. Berry, Philos. Trans. Roy. Soc. (London) 287, 237 (1977).

${ }^{21}$ P. Carruthers and F. Zachariasen, Rev. Mod. Phys. 55, 245 (1983).

${ }^{22}$ T. Takabayashi, Prog. Theor. Phys. 11, 341 (1954).

${ }^{23}$ M. S. Bartlett and E. J. Moyal, Proc. Cambridge Philos. Soc. 45, 545 (1949).

24J. P. Dahl, Physica 114A, 439 (1982).

25J. P. Dahl, in Energy Storage and Redistribution in Molecules, edited by J. Hinze (Plenum Press, New York, 1983).

${ }^{26}$ E. J. Heller, J. Chem. Phys. 67, 3339 (1977).

${ }^{27}$ H.-W. Lee and M. O. Scully, J. Chem. Phys. 77, 4604 (1982).

28J. P. Dahl and M. Springborg (unpublished).

${ }^{29}$ J. P. Dahl and M. Springborg, Mol. Phys. 47, 1001 (1982).

${ }^{30}$ J. M. Gracia-Bondía, Phys. Rev. A 30, 691 (1984).

${ }^{31}$ M. Springborg, Theor. Chim. Acta (Berlin) 63, 349 (1983).

${ }^{32}$ A. J. Thakkar, Proceedings of the Fourth International Congress in Quantum Chemistry, Uppsala, Sweden, 1982 (unpublished).

${ }^{33}$ W. Weyrich, Proceedings of the Symposium on Density Ma- trices and Density Functionals in Honour of A. John Coleman, Queen's University, Kingston, Ontario, Canada, 1985 (unpublished).

${ }^{34}$ P. A. M. Dirac, Proc. Cambridge Philos. Soc. 26, 376 (1930).

${ }^{35}$ K. Husimi, Proc. Phys. Math. Soc. Japan 22, 264 (1940).

${ }^{36}$ P.-O. Löwdin, Phys. Rev. 97, 1474 (1955).

${ }^{37}$ R. McWeeny, Rev. Mod. Phys. 32, 335 (1960).

${ }^{38}$ M. Springborg, J. Phys. A 16, 535 (1983).

${ }^{39}$ N. H. McCoy, Proc. Natl. Acad. Sci. USA 18, 674 (1932).

${ }^{40}$ J. P. Dahl, Physica Scripta 25, 499 (1982).

${ }^{41}$ A. Grossmann, Commun. Math. Phys. 48, 191 (1976).

${ }^{42}$ A. Royer, Phys. Rev. A 15, 449 (1977).

${ }^{43}$ M. Springborg, Physica 126A, 259 (1984).

44J. P. Dahl, in Semiclassical Descriptions of Atomic and Nuclear Collisions, edited by J. de Boer and J. Bang (Elsevier, Amsterdam, 1986).

${ }^{45}$ L. Cohen, J. Math. Phys. 7, 781 (1966).

${ }^{46}$ M. Springborg and J. P. Dahl, in Local Density Approximations in Quantum Chemistry and Solid State Physics, edited by J. P. Dahl and J. Avery (Plenum Press, New York, 1984).

${ }^{47}$ F. B. van Duijneveldt, IBM Research Laboratory Report No. RJ-945, 1971 (unpublished).

${ }^{48}$ S. Huzinaga (unpublished).

${ }^{49}$ R. Latter, Phys. Rev. 99, 510 (1955).

${ }^{50}$ E. H. Lieb, Rev. Mod. Phys. 53, 603 (1981).

${ }^{51}$ K. Takahashi, J. Phys. Soc. Japan 55, 76 (1986).

${ }^{52}$ M. E. Rose, Elementary Theory of Angular Momentum (John Wiley and Sons, New York, 1957). 